\title{
What's new for the clinician: excerpts from and summaries of recently published papers
}

\section{The importance of preventative maintenance in implant therapy}

FO Costa, SD Ferreira, JR Cortelli, RPE Lima, SC Cortelli, LOM Cota.

Clin Oral Invest. 2019; 23: 3161.17: 71-6.

The ability to replace teeth in a stable, predictable way has changed the way edentulous patients are treated. It has been estimated that five to 10 years after placement, $10 \%$ of implants in $20 \%$ of patients will have an inflammatory process around the devices. ${ }^{1}$ These inflammatory conditions have been classified as peri-implant mucositis and peri-implantitis.

Peri-implant mucositis (PM) is a reversible inflammatory condition whose main clinical characteristic is bleeding on probing. Erythema, swelling, and/or suppuration may also be present. Peri-implantitis (PI) is an irreversible plaqueassociated pathological condition occurring in tissues around dental implants, characterized by inflammation in the peri-implant mucosa and subsequent progressive loss of supporting bone.

Regular appointments for preventive maintenance therapy (PMT) aim to maintain the health of peri-implant tissues in the long term. ${ }^{1}$ Subgingival microbioma studies revealed that a group of bacteria, especially Tannerella forsythia, Treponema denticola, Porphyromonas gingivalis, Prevotella intermedia, Fusobacterium nucleatum, Campylobacter rectus, Prevotella nigrescens, Eubacterium nodatum, and Peptostreptococcus micros, have an important role in periodontitis (PE). ${ }^{1}$

Additionally, microbiological reviews have shown that the peri-implant microbioma, whether in health or disease, is similar to that around natural teeth. ${ }^{1}$ Costa and colleagues (2019) ${ }^{1}$ reported on a trial that sought to evaluate, in a longitudinal period of five years, the peri-implant condition and the differences in the frequencies of $T$. forsythia, $T$. denticola, P. gingivalis, P. intermedia, F. nucleatum, and Actinomyces naes/undii in individuals initially diagnosed with PM in the presence and absence of PMT.

Veerasamy Yengopal: $B C h D, B S c H o n s, M C h D, P h D$, Community Dentistry Department, School of Oral Health Sciences, University of Witwatersrand, Medical School, no. 7 York Road, Parktown 2193, South Africa.

ORCID Number: 0000-0003-4284-3367

Email: veerasamy.yengopal@wits.ac.za

\author{
ACRONYMS \\ BOPi: $\quad$ Bleeding on Probing \\ CAL: $\quad$ Clinical Attachment Level \\ GNTP group: Absence of dental visits during the \\ evaluation period \\ GTP group: At least five dental visits during the \\ evaluation period \\ PDi: $\quad$ Peri-Implant Probing Depth \\ PE: $\quad$ Periodontitis \\ PI: Peri-Implantitis \\ PLI: $\quad$ Plaque Index \\ PM: $\quad$ Peri-Implant Mucositis \\ PMT: $\quad$ Preventive Maintenance Therapy
}

\section{MATERIALS AND METHODS}

This Brazilian study took place after a five-year period during which a large task force was employed for the recruitment of the 212 initial participants who had received implant therapy.

Contacts were made through direct approach, telephone calls, telegrams, emails, and/or text messages. Eighty individuals who were diagnosed with PM at the initial examination (T1-year 2006) were recovered and underwent a new periodontal peri-implant clinical examination and micro-biological collection (T2year 2011).

These individuals were divided into two groups: one group with preventive maintenance therapy during the study period (those carrying out regular PMT with dental visits at least once a year (GTP; $n=39$ ) and another group without preventive maintenance therapy (GNTP; $n=41$ ).

In terms of clinical examinations, the following clinical parameters for four peri-implant sites in each implant were evaluated using standardized criteria: suppuration, peri-implant probing depth (PDi), bleeding on probing (BOPi), and plaque index (PLI) around all implants. 
Additionally, complete periodontal examinations were performed and included plaque index, periodontal probing depth (PD), clinical attachment level (CAL), and bleeding on periodontal probing (BOP) for four sites in each tooth.

During interviews at T2 (T2-year 2011), special attention was given to the occurrence and frequency of periodontal and peri-implant preventive maintenance within the five years following $T$ (year 2006). Frequency of PMT was determined by self-reported information and confirmed in dental records (GTP group: at least five dental visits during the evaluation period (mean $5.6 \pm 0.3$ visits); GNTP group: absence of dental visits during the evaluation period).

During PMT visits, the following procedures were performed: (1) periodontal and peri-implant status assessment, (2) application of disclosing agents and oral hygiene instructions, and (3) coronal prophylaxis and non-surgical and surgical mechanical debridement, when necessary.

Peri-implant mucositis was defined as the presence of visual inflammation and BOPi. Peri-implantitis was defined as the presence of $\mathrm{PDi} \geq 5 \mathrm{~mm}$ associated with BOPi and/or suppuration with peri-implant bone loss. Cases where the radiographs did not confirm the periimplant bone loss were diagnosed as PM.

Also, sub-gingival samples were collected at T1 and T2 in eight peri-implant sites, two in each quadrant (the peri-implant sites with the higher PDi associated with BOPi were evaluated at both times), for each individual.
Quantification of the total number of bacterial cells, A. naes/undii, P. gingivalis, T. forsythia, T. denticola, $P$. intermedia, and $F$. nucleatum was carried out by quantitative real-time polymerase chain reaction (qPCR) using TaqMan assay (TaqMan $®$ Universal PCR Master Mix II).

\section{RESULTS}

The characteristics of the sample at T1 (2006) and T2 (2011) are presented in Table 1. Individuals in the GNTP group had significantly higher values of plaque index when compared with GTP after five years $(1.9 \pm 0.5$ vs. $1.4 \pm 0.7 ; p=0.001)$.

There was a significantly higher incidence of PI in GNTP (43.9\%) than in the GTP (18\%) group. It is noteworthy that patients with $\mathrm{PI}$ in GTP, despite maintenance and necessary surgical treatment, still persisted with PI diagnosis in the final exam. All subjects $(n=12)$ who presented PM resolution at T2 were in the GTP group.

There was an increase in the number of individuals with PE in GNTP when comparing T1 $(22.0 \%)$ with T2 (41.5\%) (see Table 1).

For the bacterial analysis, in the GTP group, there was a significant decrease in Total Bacterial load, in the frequency of the bacteria analysed in the orange complex, and in the isolated frequency of $T$. forsythia, $P$. gingivalis, $P$. intermedia, and $A$. naes/undii at T2 (unadjusted and adjusted models). Additionally, there was a significant increase in the isolated frequency $F$. nucleatum (unadjusted model) at T2.

\begin{tabular}{|c|c|c|c|c|c|c|}
\hline \multirow[t]{2}{*}{ Variables } & \multicolumn{3}{|l|}{ Baseline (T1) } & \multicolumn{3}{|c|}{ Final examination (T2) } \\
\hline & GNTP $n=41$ & GTP $n=39$ & $p$ & GNTP $n=41$ & GTP $n=39$ & $p$ \\
\hline \multicolumn{7}{|l|}{ Gender $^{a}$} \\
\hline Male & 22 (53.7\%) & $24(61.5 \%)$ & 0.476 & $22(53.7 \%)$ & $24(61.5 \%)$ & 0.476 \\
\hline Female & $19(46.3 \%)$ & 15 (38.5\%) & & $19(46.3 \%)$ & $15(38.5 \%)$ & \\
\hline Age (years) ${ }^{b}$ & $22(53.7 \%)$ & $24(61.5 \%)$ & 0.476 & $22(53.7 \%)$ & $24(61.5 \%)$ & 0.476 \\
\hline \multicolumn{7}{|c|}{ Smokers/Former smokers ${ }^{a}$} \\
\hline Yes & $13(31.7 \%)$ & 8 (20.5\%) & 0.255 & $14(34.1 \%)$ & 8 (20.5\%) & 0.172 \\
\hline No & $28(68.3 \%)$ & $31(79.5 \%)$ & & 27 (65.9\%) & $31(79.5 \%)$ & \\
\hline \multicolumn{7}{|l|}{ Diabetes $^{a}$} \\
\hline Yes & $6 \quad(14.6 \%)$ & 5 (12.8\%) & 0.814 & $6(14.6 \%)$ & 7 (17.9\%) & 0.688 \\
\hline No & 35 (85.4\%) & $34(87.2 \%)$ & & $35(85.4 \%)$ & $32(82.1 \%)$ & \\
\hline \multirow[t]{2}{*}{ Number of teeth ${ }^{b}$} & 849 & 805 & 0.927 & 846 & 797 & 0.794 \\
\hline & $20.6 \pm 6.2$ & $20.6 \pm 7$ & & $20.6 \pm 6.2$ & $20.3 \pm 6.9$ & \\
\hline Average of lost teeth & $2.9 \pm 3.9$ & $4.3 \pm 5.6$ & 0.283 & $2.9 \pm 3.9$ & $3.0 \pm 4.8$ & 0.607 \\
\hline \multirow[t]{2}{*}{ Implant number ${ }^{\mathrm{b}}$} & 183 & 157 & 0.143 & 180 & 156 & 0.419 \\
\hline & $4.4 \pm 3.8$ & $3.9 \pm 2.1$ & & $4.4 \pm 3.8$ & $4.5 \pm 3.1$ & \\
\hline $\begin{array}{l}\text { Installation time of the } \\
\text { prosthesis (months) }^{\mathrm{a}}\end{array}$ & $21.3 \pm 7.1$ & $24.7 \pm 17.4$ & 0.454 & $80.5 \pm 9$ & $77.4 \pm 12.5$ & 0.457 \\
\hline Plaque index ${ }^{b}$ & $1.6 \pm 0.6$ & $1.4 \pm 0.6$ & 0.176 & $1.9 \pm 0.5$ & $1.4 \pm 0.7$ & 0.001 \\
\hline \multicolumn{7}{|l|}{ Periodontal diagnosis ${ }^{a}$} \\
\hline Healthy & 32 (78.0\%) & $29(74.4 \%)$ & 0.698 & $24(58.5 \%)$ & 28 (71.8\%) & 0.214 \\
\hline PE & 9 (22.0\%) & $10(25.6 \%)$ & & $17(41.5 \%)$ & $11(28.2 \%)$ & \\
\hline \multicolumn{7}{|l|}{ Peri-implant diagnosis ${ }^{c}$} \\
\hline Healthy & 0 & 0 & NA & $0(0.0 \%)$ & 12 (100\%) & 0.000 \\
\hline PM & 41 & 39 & NA & $23(56.0 \%)$ & 20 (51.2\%) & \\
\hline $\mathrm{PI}$ & 0 & 0 & NA & $18(43.9 \%)$ & 7 (18\%) & \\
\hline
\end{tabular}


In intra-group comparisons, there was a significant increase in Total Bacterial load, in the frequency of the bacteria analysed in the orange complex, and in the isolated frequencies of $P$. gingivalis, $P$. intermedia, and $F$. nucleatum in GNTP. There was an increase in the frequency (unadjusted model) of the red complex and A. naes/undii.

At T2, individuals diagnosed with $\mathrm{PM}$ and $\mathrm{PI}$ in the GNTP group presented a significantly higher TBL when compared with GTP. In both groups, individuals who progressed from PM to PI showed a significant increase in TBL. The GTP individuals who remained with PM showed a decrease in TBL.

\section{CONCLUSIONS}

the researchers concluded that there was a significant longitudinal increase in Total Bacterial count in the GNTP group compared with the GTP group.
Preventative maintenance therapy was shown to be beneficial in maintaining peri-implant clinical stability and homeostasis of the microbiological condition.

\section{Implications for practice:}

Preventative maintenance therapy has been shown to be crucial for total bacterial control and clinical stability of the implant. Thus, frequent recall of these patients is an important indicator of future success and functioning of the implant.

\section{Reference}

1. Costa FO, Ferreira SD, Cortelli JR. Lima RPE, Cortelli SC, Cota LOM. Microbiological profile associated with periimplant diseases in individuals with and without preventive maintenance therapy: a 5-year follow-up. Clin Oral Invest. 2019; 23: 3161.

\title{
2. Effect of a single-tufted toothbrush on the control of dental biofilm in orthodontic patients: A randomized clinical trial
}

\author{
Smaïl-Faugeron, V, Muller-Bolla, M, Sixou, J-L, Courson, F. Int J Paediatr Dent. 2019; 29: 573-84.
}

Local anaesthesia is frequently indicated in paediatric oral health care, and two techniques may be used: conventional intra-mucosal infiltration anaesthesia $(\mathrm{CIA})$, frequently used by practitioners, and intraosseous anaesthesia (IOA), which delivers the local anaesthetic within the spongy bone adjacent to the tooth to be anaesthetized.

Needle- and injection-related fears are common sources of dental anxiety in children in the case of CIA. Indeed, the needle is not only visible, but also injecting too quickly into the mucosa can lead to pain or discomfort. With IOA, the fear of the needle would be decreased because the pen grip is very close to the needle.

Also, pain during injection would be decreased because IOA can be delivered by a computerized system (QuickSleeper ${ }^{\mathrm{TM}}$ system), which delivers local anaesthesia at a constant rate and pressure.

Smail-Faugeron and colleagues $(2019)^{1}$ reported on a trial that sought to compare pain during the insertion of the needle and injection with IOA against CIA for treating first permanent molars with deep carious lesions or moderate to high severity $(\mathrm{MIH})$ lesions in children and adolescents.

Secondary objectives were to compare the two anaesthesia types in terms of pain latency, the need for additional anaesthesia and pain felt during the treatment.

\section{ACRONYMS}

CIA: Conventional Intra-Mucosal Infiltration Anaesthesia

IOA: Intraosseous Anaesthesia

MIH: Moderate to High Severity

RCT: Randomized Clinical Trial

VAS: Visual Analogue Scale

\section{METHODS}

This was a multicentre randomized clinical trial (RCT) using both a split-mouth and parallel-arm design. For the split-mouth RCT, two permanent first molars located in the same dental arch were included per patient, and for the parallel-arm RCT, only one permanent first molar was included per patient.

The following inclusion criteria were established: patients between 7 and 15 years of age; mentally and generally disease-free; cooperative (score 0 to 2 on the Venham distress scale); with one (for the parallel-arm trial) or at least two (for the split-mouth trial) first permanent molars with deep caries (i.e. dentinal lesion involving more than $50 \%$ of the entire dentin thickness evaluated by radiography) or $\mathrm{MIH}$ lesion, with preserved pulp vitality as determined by clinical and radiographic observations (treatments could be conservative or endodontic limited to pulpotomy); and without the use of analgesic drug for 48 hours before randomization. 
The exclusion criteria were periodontal alterations (periodontal pockets or dental mobility) or radiological defects (bone loss, furcation or periapical radiolucency and pre-operative clinical and radiographic observations of irreversible radicular pulpal inflammation or pulpal necrosis.

Before either type of anaesthesia was administered, a topical anaesthesia Xylocaïne ${ }^{\circledR}$ visqueuse $2 \%$ was applied for one to two minutes on previously dried mucosa. For CIA, a 16-mm-long needle was used to inject in the maxillary region and a 35-mm-long needle was used for mandibular anaesthesia.

IOA was carried out using the QuickSleeperTM system followingthethree-step procedure described bythemanufacturer. The anaesthetic solution used in both techniques was 4\% articaine with 1:200 000 adrenalin.

All outcomes were recorded on the day of the intervention. The primary outcome measure was pain felt during the insertion of the needle and injection of the anaesthetic, assessed at the end of the anaesthesia, with a Visual Analogue Scale (VAS) ranging from $0 \mathrm{~cm}$ (no pain) to $10 \mathrm{~cm}$ (very much pain).

Three secondary outcomes were evaluated: latency, the need for additional anaesthesia during the treatment and pain felt during the treatment. The latency was assessed examining the sensitivity of the vestibular sulcus for CIA or lingual sulcus for IOA by using a probe at the end of the injection (an exam was conducted every minute until the sulcus was insensitive to the probe). Patient assessed the pain felt during the treatment, recording on the VAS at the end of the dental treatment session.

For anaesthesia allocation, a computer-generated, permuted-block randomization sequence was used for the parallel arm RCTs. For the split-mouth RCT, one permanent first molar was randomly allocated to one of the techniques (e.g., IOA) and the other permanent first molar belonging to the same dental arch in the same child was allocated to the other technique (e.g., CIA).

A 7- to 21-day interval was established between the two procedures. For the parallel-arm RCT, one patient with one permanent molar first was randomly allocated to one of the techniques (IOA or CIA). Patients - evaluators - were unaware of which of the two techniques was used, whereas practitioners were not.

\section{RESULTS}

For each patient enrolled in the split-mouth RCT, about five were enrolled in the parallel-arm RCT, which allowed for not losing any eligible patients. In total, 158 children aged 7-15 years for whom parental consent had been obtained were deemed eligible for inclusion.

Of these, 30 (mean age: 9.0 years, SD: 2.3) participated in the split-mouth RCT and 128 (mean age: 10.4 years, SD: 2.5) in the parallel-arm RCT. For the parallel-arm $\mathrm{RCT}, 63$ patients were allocated to the IOA group and 65 to $\mathrm{ClA}$ group.

\section{Split-mouth RCT results}

The mean VAS scores were $0.73 \pm 1.31 \mathrm{~cm}$ for the $1 \mathrm{OA}$ and $1.43 \pm 1.45 \mathrm{~cm}$ for the CIA groups. The mean $(95 \%$ $\mathrm{Cl})$ for the difference in paired proportions was $-0.70 \pm$

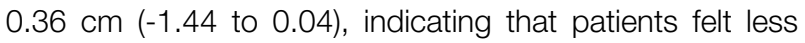
pain on the insertion of the needle and the injection with IOA compared with $\mathrm{CIA}$, but this difference was not statistically significant $(P=0.06)$.

The mean latency was $1.07 \pm 0.25$ minutes for the IOA and $2.83 \pm 2.64$ minutes for the CIA groups. The mean $(95 \% \mathrm{Cl})$ for the difference in paired proportions was $-1.77 \pm 0.51(-2.77$ to -0.77$)$, indicating that latency was statistically decreased with IOA compared with CIA with a statistically significant difference $(P=0.001)$.

For pain felt during treatment, the mean VAS scores were $1.07 \pm 1.76 \mathrm{~cm}$ for the IOA and $0.53 \pm 0.82 \mathrm{~cm}$ for the CIA groups. The mean $(95 \% \mathrm{Cl})$ for the difference in paired proportions was $0.53 \pm 0.37 \mathrm{~cm}(-0.19$ to 1.25), indicating that patients felt less pain during treatment with CIA compared with IOA, but this difference was not statistically significant $(P=0.14)$.

\section{Parallel-arm RCT results}

The mean VAS scores were $1.17 \pm 1.40 \mathrm{~cm}$ for the IOA and $1.86 \pm 1.81 \mathrm{~cm}$ for the CIA groups. The mean $(95 \%$ $\mathrm{Cl})$ for the difference in paired proportions was -0.69 $\pm 0.29 \mathrm{~cm}(-1.25$ to -0.12$)$, indicating that patients felt less pain on the insertion of the needle and the injection with IOA compared with CIA, with a statistically significant difference $(P=0.02)$.

The mean latency was $1.63 \pm 0.97$ min for the $1 O A$ and $3.08 \pm 2.11 \mathrm{~min}$ for the ClA groups. The mean $(95 \% \mathrm{Cl})$ for the difference in paired proportions was $-1.44 \pm 0.29$ $(-2.02$ to -0.87$)$, indicating that latency was statistically decreased with IOA compared with CIA with a statistically significant difference $\left(P=2.87 .10^{-6}\right)$.

For pain felt during the treatment, the mean VAS scores were $0.90 \pm 1.51 \mathrm{~cm}$ for the IOA and $0.88 \pm 1.64 \mathrm{~cm}$ for the CIA groups.

The mean $(95 \% \mathrm{Cl})$ for the difference in paired proportions was $0.03 \pm 0.28 \mathrm{~cm}(-0.52$ to 0.58$)$, indicating that patients felt less pain during treatment with CIA compared with IOA, but this difference was not statistically significant $(P=0.92)$.

\section{Combined treatment effect from split-mouth and parallel-arm RCTs}

For all outcomes, findings from the two types of trials were consistent: for pain felt during insertion of the needle and injection, $p$ value was equal to 0.98 ; for latency, $p$ value was equal to 0.58 ; for need for additional anaesthesia, $p$ value was equal to 0.66; and for pain felt during treatment, $p$ value was equal to 0.27 .

Hence, there was no significant difference for all of these variables when the results of the two types of study designs were combined. 


\section{CONCLUSIONS}

For all outcomes, findings from the two types of trials were consistent: for pain felt during insertion of the needle and injection, latency, the need for additional anaesthesia and for pain felt during treatment, there were no signiicant differences between the two techniques (IOA vs CIA).

\section{Implications for practice}

The evidence for computerised injection systems suggest that they are still not superior to the conventional methods of administering local anaesthesia for the outcomes that were assessed in this study.

\section{Reference}

1. Smail-Faugeron, V, Muller-Bolla, M, Sixou, J-L, Courson, F. Evaluation of intraosseous computerized injection system (QuickSleeper ${ }^{\mathrm{TM}}$ ) vs. conventional infiltration anaesthesia in paediatric oral health care: A multicentre, single-blind, combined split-mouth and parallel-arm randomized controlled trial. Int J Paediatr Dent. 2019; 29: 573-84.

\section{Do the CPD questionnaire on page 405}

The Continuous Professional Development (CPD) section provides for twenty general questions and five ethics questions. The section provides members with a valuable source of CPD points whilst also achieving the objective of CPD, to assure continuing education. The importance of continuing professional development should not be underestimated, it is a career-long obligation for practicing professionals.

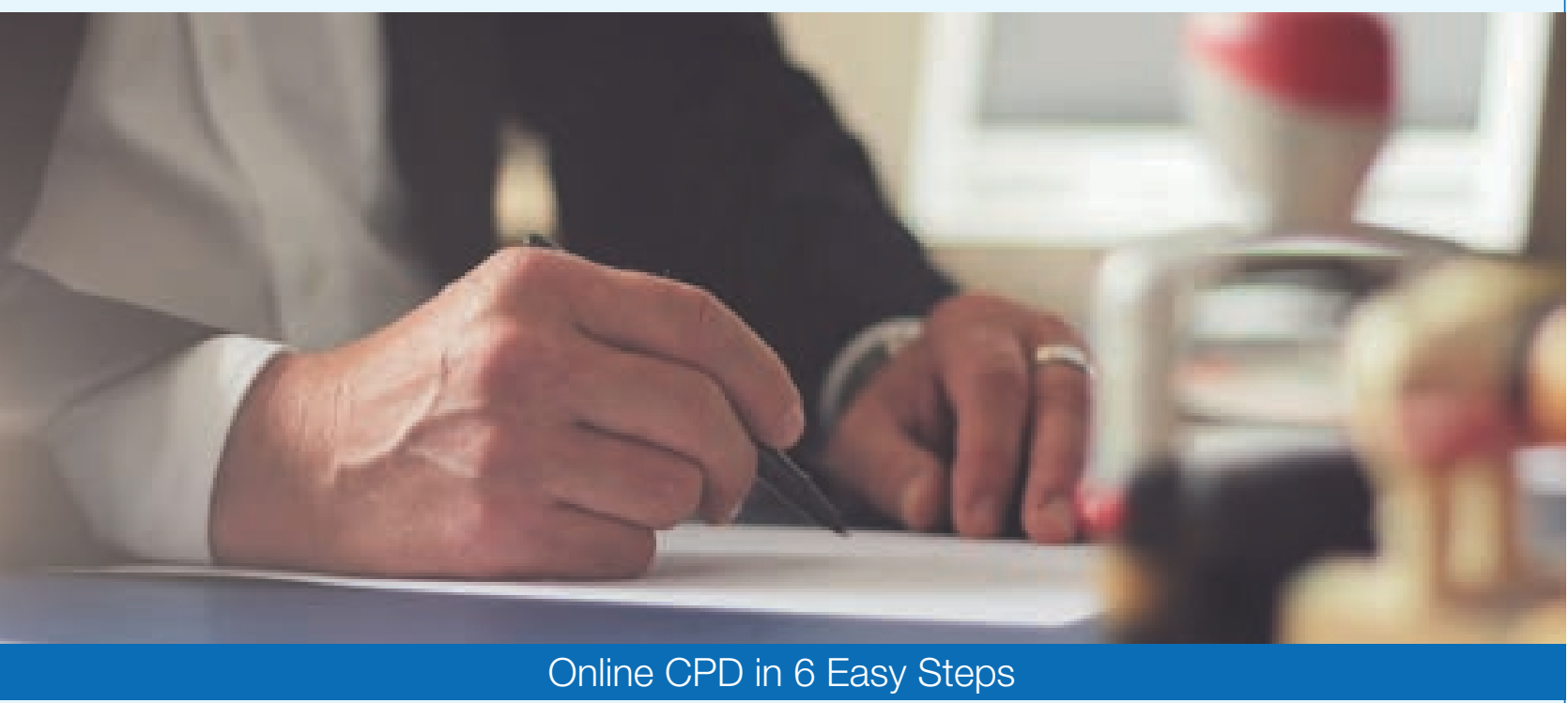

1 Go to the SADA website www.sada.co.za.

2 Log into the 'member only' section with your unique SADA username and password.

3 Select the CPD navigation tab.

4 Select the questionnaire that you wish to complete.

5 Enter your multiple choice answers. Please note that you have two attempts to obtain at least $70 \%$.

6 View and print your CPD certificate. 